\title{
Effect of carrier transfer on the PL intensity in self-assembled In (Ga) As/GaAs quantum rings
}

\author{
W. Ouerghui ${ }^{1, a}$, J. Martinez-Pastor ${ }^{2}$, J. Gomis ${ }^{2}$, A. Melliti ${ }^{1}$, M.A. Maaref ${ }^{1}$, D. Granados ${ }^{3}$, and J.M. Garcia ${ }^{3}$ \\ 1 Unité de Recherche des Physiques des Semiconducteurs et Capteurs, Institut Préparatoire aux Études Scientifiques \\ et Techniques, La Marsa 2070, Tunis, Tunisia \\ 2 Instituto de Ciencia de los Materials, Universidad de Valencia, PO Box 22085, 46071 Valencia, Spain \\ 3 Instituto de Microelectrónica de Madrid, Isaac Newton 8, 28760 Tres Cantos, Madrid, Spain
}

Received: 9 May 2006 / Accepted: 9 June 2006

Published online: 23 August 2006 - (c) EDP Sciences

\begin{abstract}
We present results concerning the carrier transfer between $\operatorname{In}(\mathrm{Ga})$ As quantum rings in a stacked multilayer structure, which is characterised by a bimodal size distribution. This transfer of carriers explains the observed temperature behaviour of diode lasers based on that kind of stacked layer structures. The inter-ring carrier transfer can be possible by phonon assisted tunnelling from the ground state of the smallring family towards the big-ring family of the bimodal size distribution. This process is thermally activated in the range $40-80 \mathrm{~K}$.
\end{abstract}

PACS. 78.55.Cr Cr-III-V semiconductors - 78.67.Pt Multilayers; superlattices - 78.47.+p Time-resolved optical spectroscopies and other ultrafast optical measurements in condensed matter

\section{Introduction}

Stacking of self-assembled quantum dots (QD) in multilayer structures has been proposed as an efficient mechanism to improve the performance of new devices based on them. Many approaches to control the QD size uniformity have been introduced in order to improve the laser performance parameters: lower threshold current, temperatureinsensitive operation, larger modulation bandwidth, lower chirp and linewidth enhancement factor [1-6]. A precise control over the QD height, width and shape is of crucial importance, since the geometry has a clear influence on the quantum confinement of the charge carriers and hence determines QD optical properties. In this sense, some strategies have been recently developed to change the shape and size of InAs QD during the growth of the InAs layer and subsequent capping with a thin barrier material of GaAs [7-9]. Following this method, quantum rings $(\mathrm{QR})$ can be obtained, which exhibit interesting basic optical properties [10]. Furthermore, QRs are potential candidates for the development of laser diodes at $980 \mathrm{~nm}$ at room temperature and high-speed optoelectronic devices. In reference [8], the study of a semiconductor laser structure is reported. It incorporates in the intrinsic active region three QR stacked layers separated by $10 \mathrm{~nm}$ thick GaAs spacers. This spacer ensures well-developed

\footnotetext{
a e-mail: ouerghuiwalid@yahoo.fr
}

rings with optical emission like that of single layers. The multimodal stimulated emission is centred on $930 \mathrm{~nm}$ at $77 \mathrm{~K}$ and it has a minimum threshold current density per QR layer of $69 \mathrm{~A} \mathrm{~cm}^{-2}$ [8], which is not a bad value.

The aim of this work is to understand the exciton recombination dynamics at low and high temperatures in such a kind of QR multilayer structures. The study has been carried out by means of steady state and time resolved photoluminescence experiments, which have been analysed by using a rate equation model that includes the carrier transfer between the observed two QR families (each one characterized by a defined height) representative of the ensemble. A precedent of the observed phenomenology (carrier transfer in vertical stacks) is reported for an intentionally grown double layer QD system, with two very different size distributions [11-13]. Besides, this kind of phenomenology can positively used for laser engineering purposes, as suggested in reference [13].

\section{Experiment}

The samples under study have been grown by molecular beam epitaxy (MBE). The QRs were obtained after a growth interruption when the dots were partially covered with $2 \mathrm{~nm}$ of GaAs under a flux of $\mathrm{As}_{2}$, because of a balance between InGaAs alloying and InAs dewetting processes [14]. More details about the growth of these kinds 


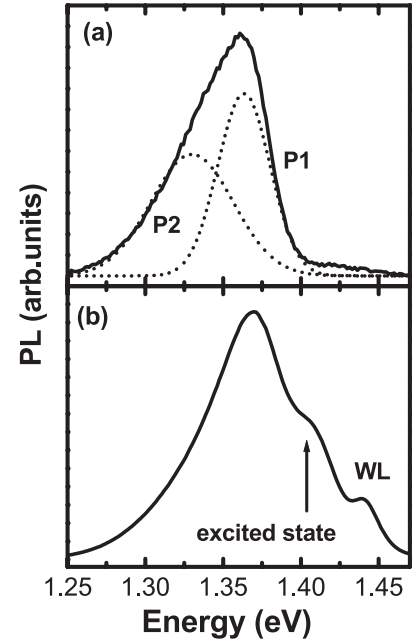

Fig. 1. Low-temperature PL spectra of self-organised InGaAs/GaAs QR excited at $2.43 \mathrm{eV}$ : (a) low density excitation $\left(3 \mathrm{~W} / \mathrm{cm}^{2}\right)$, (b) high density excitation $\left(300 \mathrm{~W} / \mathrm{cm}^{2}\right)$. The contribution of the excitonic excited state and wetting layer recombination are indicated in this figure. Dashed lines correspond to the best two-Gaussian fit of the measured PL band.

of samples can be found in references $[8,15,16]$. The two samples studied here contain three layers of QR stacked by using different GaAs spacer (added after the QR formation to cap them) thicknesses: 14 (sample A) and $1.5 \mathrm{~nm}$ (sample B). In each layer, the total amount of deposited InAs was just above the critical thickness (1.6 ML) necessary for the $3 \mathrm{D}$ transition to take place and obtain very low in-plane densities of QR nanostructures. The typical size of the original dots was around 20-40 nm diameter and around $11 \mathrm{~nm}$ of height, in average. The presence of QR nanostructures in these stacks, and their shape and composition peculiarities, has been revealed and studied by Atomic Force Microscopy (AFM), Transmission Electron Microscopy (TEM) [15] and Scanning Tunneling Microscopy (STM) [17].

The continuous wave PL experiments were performed by using the $514 \mathrm{~nm}$ line of and $\mathrm{Ar}^{+}$laser as excitation source. The PL signal was dispersed by a monochromator and synchronously detected with a cooled Ge detector. In time resolved experiments, the excitation was done at $750 \mathrm{~nm}$ by a green $\mathrm{Nd}: \mathrm{YVO}_{4}$ pumped mode locked Ti:Sapphire laser (Mira 900D, Coherent), providing 2 ps pulses at a repetition rate of $76 \mathrm{MHz}$. The PL signal was dispersed by a single $0.5 \mathrm{~m}$ focal length imaging spectrograph and detected by a synchroscan streak camera (Hamamatsu C5680) with a type $\mathrm{S}_{1}$ cooled photocathode. The overall time response of the system in the widest temporal window (about $2 \mathrm{~ns}$ ) was around $40 \mathrm{ps}$ (full width at half maximum). In both kinds of experiments the samples were held in the cold finger of a closed-cycle cryostat to vary the temperature in the range $12-300 \mathrm{~K}$, approximately.

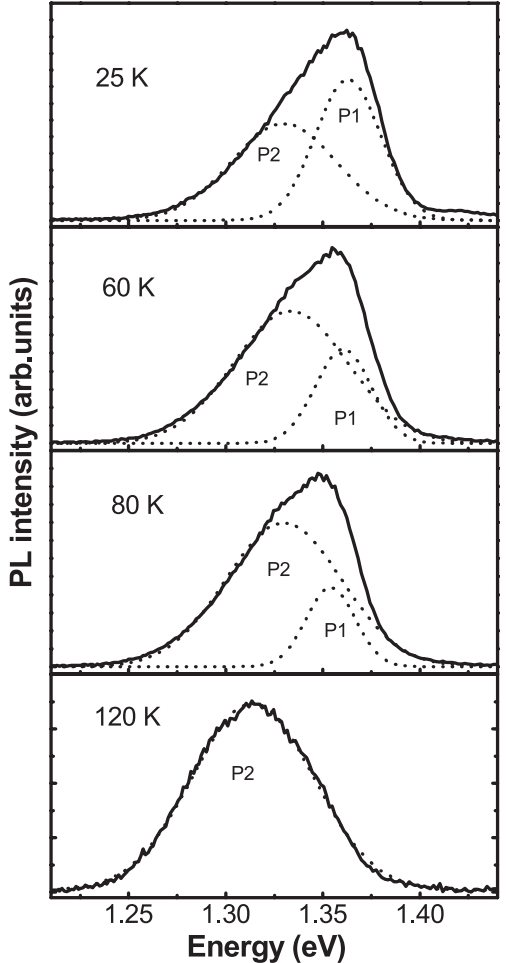

Fig. 2. PL spectra at different temperature for sample A. Dashed lines correspond to PL-Gaussian components (P1-P2) at the different temperatures.

\section{Results and discussion}

Figure 1a shows the PL spectrum of sample A under low excitation density conditions $\left(3 \mathrm{~W} / \mathrm{cm}^{2}\right)$, just around one photogenerated electron-hole per QR, because the QR sheet density is relatively low $\left(2-5 \times 10^{9} \mathrm{~cm}^{-2}\right)$. This condition ensures that emission arises from the optically active ground state of the QR ensemble. The PL peak energy occurs at $1.361 \mathrm{eV}$ and the PL shape cannot be reproduced by a simple Gaussian. At least two Gaussian contributions peaked at 1.320 and $1.361 \mathrm{eV}$ are needed to reproduce the PL band, as observed in Figure 1a (dotted curves). We attribute the measured PL band to a bi-modal size distribution of the QR ensemble (i.e., two different size families), being similar in each of the three stacked layers in average. Increasing the excitation density $\left(300 \mathrm{~W} / \mathrm{cm}^{2}\right)$, a new contribution to the PL band appears at around 35-40 meV above the PL peak energy, as shown in Figure 1b. This energy difference is consistent with the first excitonic excited state ( $p$-like state) of the QR ensemble [18]. Furthermore, the observation of this contribution and that of the wetting layer [WL in Fig. 1b] reinforces the interpretation of $\mathrm{P} 1-\mathrm{P} 2$ like ground state recombination of different size QR families in the ensemble.

PL spectra at different selected temperatures within the range of interest are shown in Figure 2. The two Gaussian contributions (P1-P2) representative of the QR ensemble are followed with increasing the temperature, as indicated in Figure 2. Above $120 \mathrm{~K}$ the PL band is 


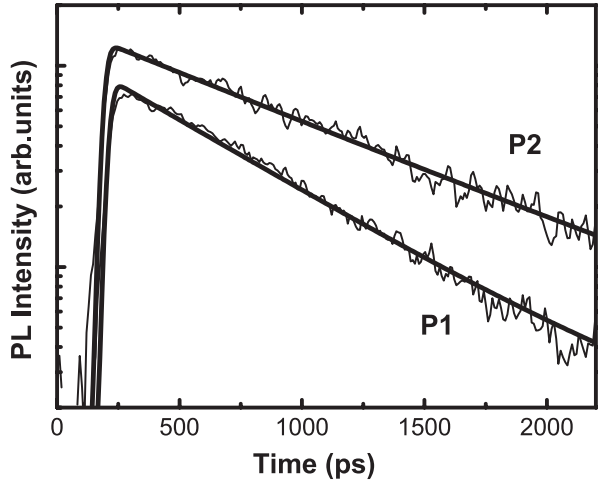

Fig. 3. Photoluminescence transients at $10 \mathrm{~K}$ for both populations (P1and P2) measured under low excitation density $\left(4 \mathrm{~W} / \mathrm{cm}^{2}\right)$ in sample $\mathrm{A}$ and the best single exponential fits (continuous lines).

practically symmetrical (single Gaussian), because of the negligible influence of P2 on its low energy side.

Figure 3 shows the PL transients measured at $10 \mathrm{~K}$ for the detection energy corresponding to high and low energy peaks, P1 and P2, respectively. The measured PL transients exhibit a mono-exponential decay, under low excitation conditions, as shown in Figure 3. The PL decay times deduced from those transients are 610 and 880 ps for P1 and P2 components, respectively. These values suggests that the PL arises from QR ground states, because the excited state recombination is typically faster and sometimes bi-exponential PL transients are measured [19,20].

Figure 4 shows the Arrhenius plot of the integrated PL intensity for both $\mathrm{P} 1$ and $\mathrm{P} 2$ components in sample A (14 nm spacer) under low excitation density conditions. We can clearly distinguish two different temperature regions. Below $40 \mathrm{~K}$, The PL band and its integrated intensity does not change. From 40 to $80 \mathrm{~K}$, the low-energy side of the emission band (P2) gains in intensity with respect to high energy part (P1), as shown in Figure $4 \mathrm{~b}$. The integrated intensity of $\mathrm{P} 1$ decreases with temperature, as observed in Figure 4a, by exhibiting an activated behaviour, according to the expression [21]:

$$
I_{1}(T) \sim \frac{I_{1}(0)}{\left[1+a_{1} e^{-\frac{E_{1}}{k T}}\right]}
$$

where $E_{1}$ is the activation energy of the thermal carrier escape out of $\mathrm{P} 1$-family rings and $a_{1}$ is the corresponding rate ratio between the radiative and escape mechanisms. The best fitting of the experimental results to equation (1) is shown in Figure $4 \mathrm{a}$ as a continuous line, by using $E_{1}=30 \pm 5 \mathrm{meV}$. This value is close to the GaAs (or InAs confined) LO phonon energy, but also to the energy difference between $\mathrm{P} 1$ and $\mathrm{P} 2$ ring families (their average ground status). The value of $a_{1}$ is close to 250 , even if its relative error is as high asd $20-30 \%$. It is worth noting that the temperature at which the PL intensity of $\mathrm{P} 2$ begins to increase (i.e., the process of carriers transfer between QR families begin), $40 \mathrm{~K}$, is much smaller than that found for QD $(T \geq 160 \mathrm{~K})[11]$. The most likely transfer mechanism,

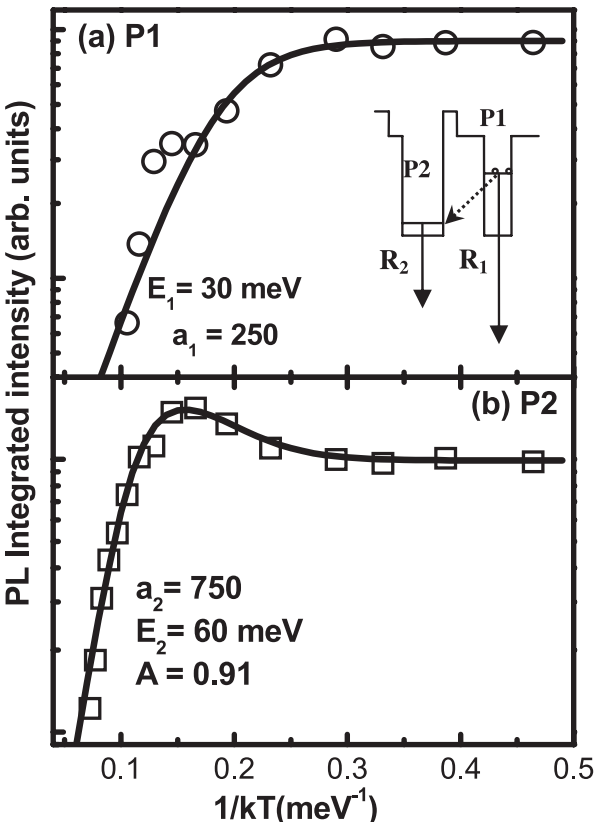

Fig. 4. Arrhenius plot of the PL integrated intensity for P1 (a) and P2 (b) QR-family. The best fits to equation (1), (for P1 population) and 4 (for P2 population) are represented by continuous lines.

phonon assisted tunneling, as suggested in reference [11], is thus significantly stronger for QR. The main differences in the carrier transfer between $\mathrm{QR}$ and $\mathrm{QD}$ are possibly associated to the large in-plane dimensions of QR (of the order of $100 \times 100 \mathrm{~nm}^{2}$ ) heterostructures [7].

The above given equation (1) cannot account for the temperature evolution of the PL integrated intensity for P2 family, because it increases in the range $40-80 \mathrm{~K}$, and decreases again above $80 \mathrm{~K}$, as shown in Figure $4 \mathrm{~b}$. The observed phenomenology suggests the existence of a thermal activated carrier transfer mechanism from P1 towards $\mathrm{P} 2$ rings, as indicated in the inset of Figure 4a. The origin of such inter-ring (P1-P2) carrier transfer would be a nonresonant tunneling process assisted by optical phonons, as suggested in references [11-13]. The charged particles to tunnel out from $\mathrm{P} 1$ towards $\mathrm{P} 2$ rings are more probably electrons, given their lighter effective mass. The rate equation model representing our system is similar to that reported in reference [22]:

$$
\begin{aligned}
\frac{d n_{1}(T)}{d t}= & 0=g N_{1}-n_{1}(T) U_{1} e^{-\frac{E_{1}}{k T}}-n_{1}(T) R_{1} \\
\frac{d n_{2}(T)}{d t}= & 0=g N_{2}+n_{1}(T) U_{1} e^{-\frac{E_{1}}{k T}} \\
& -n_{2}(T) U_{2} e^{-\frac{E_{2}}{k T}}-n_{2}(T) R_{2}
\end{aligned}
$$

where

(1) $g N_{1}$ and $g N_{2}$ represent the number of charge carriers captured per second at $0 \mathrm{~K}$ in the $\mathrm{P} 1$ and $\mathrm{P} 2 \mathrm{QR}$ families, respectively.

(2) $R_{1}$ and $R_{2}$ are the radiative recombination rates of $\mathrm{P} 1$ and P2 QR populations. 
(3) $U_{1(2)}(T)=U_{1(2)} e^{-\frac{E_{1(2)}}{k T}}$ is the thermal escape rate of carriers out of P1 (P2) QR, characterized by the activation energy $E_{1(2)}$.

For steady state conditions, the PL intensity associated to $\mathrm{P} 1$ family, $I_{1}(\mathrm{~T})$, is that given by equation (1) (with $\left.I_{1}(0)=g N_{1} / R_{1}\right)$, and $I_{2}(\mathrm{~T})$ will be:

$$
I_{2}(T)=\frac{I_{2}(0)}{\left[1+a_{2} e^{-\frac{E_{2}}{k T}}\right]}\left[1+\frac{A}{1+\frac{1}{a_{1}} e^{\frac{E_{1}}{k T}}}\right]
$$

where $I_{2}(0)=g N_{2} / R_{2}, A=N_{1} / N_{2}$ is the ratio between $\mathrm{QR}$ densities of $\mathrm{P} 1$ and $\mathrm{P} 2$ families, which can be independently deduced from the Gaussian fits in Figure $1(A=$ 0.91 at the lowest temperature), and $a_{1(2)}=U_{1(2)} / R_{1(2)}$. The radiative rates for $\mathrm{P} 1$ and $\mathrm{P} 2, R_{1}$ and $R_{2}$, will be given by the inverse of the time decays at the lowest temperature (Fig. 3), $R_{1}=1.6 \times 10^{9} \mathrm{~s}^{-1}$ and $R_{2}=1.13 \times 10^{9} \mathrm{~s}^{-1}$. In this way, the values of $U_{1(2)}$ and $E_{1(2)}$ can be deduced from the best fits of the experimental variation of $I_{1(2)}(T)$ to equation (14), as represented by continuous lines in Figure 4 . The parameters used in the fit of $I_{1}(T)$ nicely reproduce the experimental increase of $I_{2}(T)$ in the 40-80 K temperature region, as observed in Figure $4 \mathrm{~b}$, whereas the high temperature region (not complete in this study) is reproduced by using $E_{2}=60 \pm 10 \mathrm{meV}$ and $a_{2}=750$. The best fitting values for P1-P2 and P2-WL transfer rates are $U_{1}=4 \times 10^{11} \mathrm{~s}^{-1}$ and $U_{2}=8.5 \times 10^{11} \mathrm{~s}^{-1}$. The relatively small value of $U_{1}$ can be reasonable for a transfer rate like the proposed one, based on thermal assisted tunneling from P1 to P2 QR. On the contrary, the value of $U_{2}$ is smaller than expected for QD-WL thermionic transfer of (correlated) carriers [23]. Something similar occurs for $E_{2}$, nearly half the energy difference from the P2 QR ground state to the WL level. The origin of this discrepancy can be the escape of carriers from the first exciton excited state (P-shell) of the P2 family, given that a certain thermal population (the energy separation was around $35 \mathrm{meV}$ ) of this shell is possible above $80-100 \mathrm{~K}$, more if the excitation density was not really low. A second and less intrinsic origin could be the existence of interface defects between the $\mathrm{QR}$ and the GaAs barriers, given the large surface of these nanostructures and the fact that we have three stacked layers. It seems to be reasonable to assume that the quantity of impurities or defect incorporated in stacked multi-layer structures would be larger than in single or double layers ones because of the accumulated stress in the first case. In any case, the thermionic emission is not the main scope of this work, because it is better know in self-assembled QD.

A more direct evidence of the carrier transfer mechanism between $\mathrm{P} 1$ and $\mathrm{P} 2$ rings would be given by the temperature evolution of the time decays for P1(2) population, $\tau_{1(2)}$, as shown in Figure 5 . For the model depicted above we have:

$$
\begin{aligned}
& \tau_{\mathrm{DECAY}}^{-1}(1)=\tau_{1}^{-1}=R_{1}+U_{1} e^{-\frac{E_{1}}{k T}} \\
& \tau_{\mathrm{DECAY}}^{-1}(2) \sim \tau_{2}^{-1}=R_{2}+U_{2} e^{-\frac{E_{2}}{k T}} .
\end{aligned}
$$

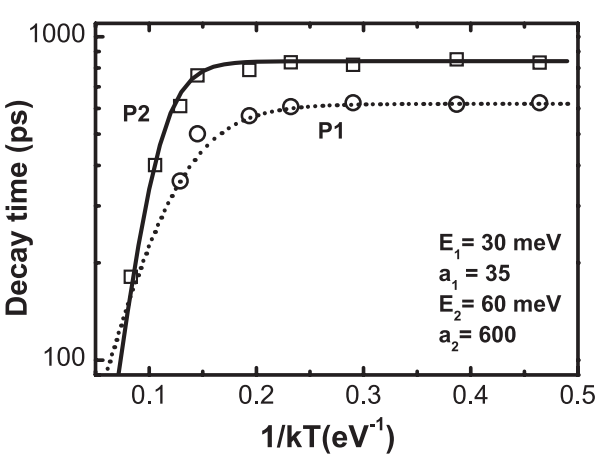

Fig. 5. Temperature evolution of the decay time for $\mathrm{P} 1$ and P2 populations. Dashed and continuous lines are the best fits to equations (5) and (6), respectively.

The time decay of the P2 population, $\tau_{\mathrm{DECAY}}(2)$ is approximately $\tau_{2}$, because of the small influence of the carrier transfer between $\mathrm{P} 1$ and $\mathrm{P} 2 \mathrm{QR}$ to the transient behaviour of the latter population. The temperature region in which TRPL was measured (in order to maintain the same low excitation conditions) is more restricted than that represented in Figure 4, because of the PL signal quenching, especially for the P1 QR family. In any case, the experimental variation of the time decays at P1-P2 detection energies is nicely reproduced by equations $(5,6)$, respectively, as shown in Figure 5 (dot and continuous lines). The activation energies found for steady state conditions seem to hold for the temperature evolution of the time decay, demonstrating the validity of the model presented here, even if the excitation energy in the TRPL experiments was notably different. This can be the reason for the apparent (we have a very restricted temperature region of variation) factor 10 difference in $a_{1}$ (and hence $U_{1}$ ) from steady state to pulsed conditions. For $U_{2}$ we find a value very close in both cases, within the experimental errors.

The study of sample B (1.5 nm spacer) serves to differentiate the effect of reducing the spacer thickness on the lateral tunneling mechanism. Figure 6 shows the PL spectra at different temperatures for this sample. The main observed differences between samples A and B are: (i) it is observed a simultaneous decrease of the PL intensity for the two Gaussian components, P1 and P2, of the QR ensemble; (ii) the linewidth of the high energy component is particularly narrow, $23 \mathrm{meV}$ in the case of sample B, which is a measure of a slight increase of quality from thick $(14 \mathrm{~nm})$ to narrow GaAs spacer $(1.5 \mathrm{~nm})$ of the QR layers. In a more complete study carried out to study the influence of the electronic coupling between QR in the different layers, we concluded that in sample B the tunneling effect is very important and it is a mechanism opened at the lowest measured temperatures.

\section{Conclusion}

We have presented measurements of the temperature dependence of the integrated PL intensity in quantum rings, 


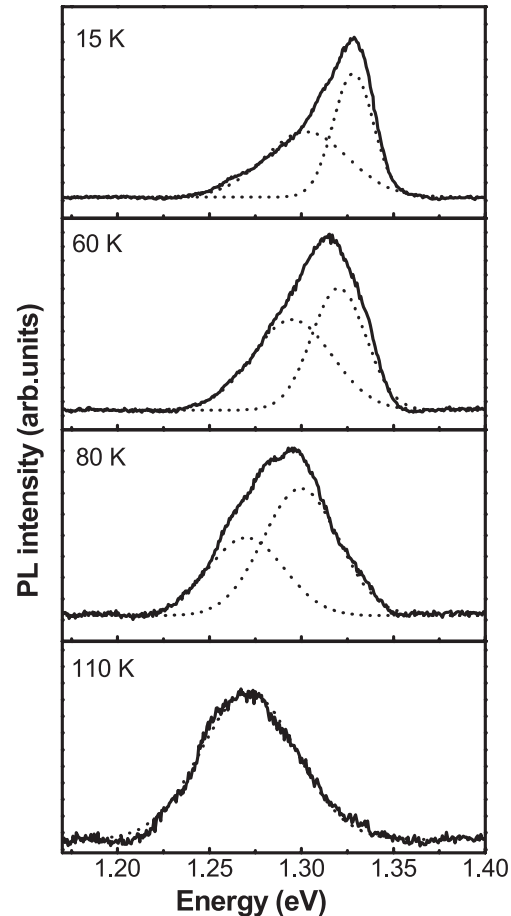

Fig. 6. PL spectra at different temperatures for sample B and their corresponding P1 and P2 Gaussian components (dashed lines).

which are explained qualitatively in terms of a coupled rate equation system, including the effect of lateral carriers transfer between rings (from P1-rings toward P2-ones). The tunneling effect between the two size distributions is found to be thermally activated between 40 and $80 \mathrm{~K}$. The assistance of optical phonons to facilitate such a lateral tunneling can be the origin of the observed thermal activation for this mechanism. The temperature evolution of the time decay measured for $\mathrm{P} 1$ and $\mathrm{P} 2$ populations are also consistent with this electron transfer mechanism between QR of different sizes. From the application point of view, the presence of such a high lateral ring-ring coupling in the QR ensemble can be the origin of the important increase of the threshold current characteristic temperature $T_{\mathrm{o}}$, which have been recently reported in laser diodes for this kind of QR ensembles [8].

This work was partially supported by Spanish MCyT Nanoself I and II projects TIC2002-04096-C03 and TEC2005-05781C03-03, the SANDiE Network of excellence (Contract No. NMP4-CT-2004-500101) and the AECI Spain-Tunisia bilateral research action No. 2/04/R.

\section{References}

1. D.L. Huffaker, G. Park, Z. Zou, O.B. Shchekin, D.G. Deppe, Appl. Lett. 73, 2566 (1998)

2. Z.-G. Wang, Y.-H. Chen, F.-Q. Liu, B. Xu, J. Cryst. Growth 227, 228, 1132 (2001)

3. R.L. Sellin, C. Ribbat, D. Bimberg, F. Rinner, H. Konstanzer, M.T. Kelemen and M. Mikulla, Electron. Lett. 38, 883 (2002)

4. S. Pradhan, S. Ghosh, P. Bhattacharya, Electron. Lett. 38, 1449 (2002)

5. O.B. Shchekin, D.G. Deppe, Appl. Phys. Lett. 80, 3277 (2002)

6. L. Zhang, Th.F. Boggess, Appl. Phys. Lett. 76, 1222 (2000)

7. J. Garcia, G. Medeiros Ribeiro, K. Schmidt et al., Appl. Phys. Lett. 71, 2014 (1997)

8. F. Suarez, D. Granados, M.L. Dotor, J.M. García, Nanotechnology 15, S126 (2004)

9. D. Granados, J.M. García, Appl. Phys. Lett. 82, 2401 (2003)

10. K. Karrai, R.J. Warburton, C. Schulhauser, A. Höegele, B. Urbaszek, E.J. McGhee, A.O. Govorov, J.M. García, B.D. Gerardot, P. M. Petroff, Nature 427, 135 (2004)

11. Yu. I. Mazur, X. Wang, Z.M. Wang, G.J. Salamo, M. Xiao, H. Kissel, Appl. Phys. Lett. 81, 2469 (2002)

12. Atsushi Takeuchi, Takamasa Kuroda, Kazuo Mase, Yoshiaki Nakata, Naoki Yokoyama, Phys. Rev. B 62, 1568 (2000)

13. T. Nakaoka, J. Tatebayashi, Y. Arakawa, T. Saito, J. Appl. Phys. 96, 150 (2004)

14. M.L. Dotor, M. Recio, D. Golmayo, F. Briones, J. Appl. Phys. 72, 5861 (1992)

15. D. Granados, J.M. García, T. Ben, S.I. Molina, Appl. Phys. Lett. 86, 071918 (2005)

16. J. Garcia, G. Medeiros Riberio, K. Schmidt et al., Appl. Phys. Lett. 71, 2014 (1997)

17. P. Offermans, P.M. Koenraad, J.H. Wolter, D. Granados, J.M. García, V.M. Fomin, V.M. Gladilin, J.T. Devreese, Appl. Phys. Lett. 87, 1 (2005)

18. B. Alén, J. Martínez-Pastor, D. Granados, J.M. García, Phys. Rev. B 72, 155331 (2005)

19. H. Yu, S. Lycett, C. Roberts, R. Murray, Appl. Phys. Lett. 69, 4087 (1996)

20. R. Heitz, A. Kalburge, Q. Xie, M. Grundmann, P. Chen, A. Hoffmann, A. Madhukar, D. Bimberg, Phys. Rev. B 57, 9050 (1996)

21. R. Heitz, I. Makhametzanov, A. Madhukar, A. Hoffmann, D. Bimberg, J. Electron. Mater. 28, 520 (1999)

22. G. Saint-Girons, I. Sagnes, J. Appl. Phys. 91, 10115 (2002)

23. S. Sanguinetti, M. Henini, M. Grassi Alessi, M. Cappizi, P. Frigeri, S. Franchi, Phys. Rev. B 60, 8276 (1999) 